\title{
Variables preoperatorias e intraoperatorias asociadas al aumento del tiempo quirúrgico en la exodoncia de terceros molares inferiores
}

\section{Pre-surgical and surgical factors related to increase of surgical time in lower third molar remove}

\author{
Olate $\mathrm{S}^{*}$, Alister JP**, Alveal R***, Soto $\mathrm{M}^{*}$, de Miranda Chaves Netto HD****, \\ Thomas D*
}

\section{RESUMEN}

Introducción: La exodoncia de tercer molar es un procedimiento frecuente en odontología, lo que exige conocer los factores que pueden anticipar el tiempo quirúrgico y sus características. El objetivo de esta investigación es conocer los factores asociados al aumento del tiempo quirúrgico (ATQ) en la exodoncia de terceros molares inferiores (3M).

Material y método: 145 sujetos de entre 15 y 40 años fueron seleccionados desde tres centros quirúrgicos de Temuco (Chile), en los que fueron realizados 158 cirugías; todos los pacientes consultaron por exodoncia de tercer molar siendo operados en tales centros. Las variables del paciente, del diente y de la cirugía fueron estudiadas; la información fue analizada con el paquete estadístico Stata 9.1, empleando en test de Fisher y chi cuadrado con $\mathrm{p}<0,05$ para establecer significado estadístico.

Resultados: el tiempo quirúrgico para cada cirugía fue menor de 20 minutos en el $50 \%$ de los casos y menor a 30 minutos en el $75 \%$ de los casos. La edad del paciente, la posición del tercer molar, la presencia de pericoronaritis y la anatomía desfavorable de las raíces de $3 \mathrm{M}$ fueron significativamente asociadas con ATQ $(p<0,05)$; la realización de colgajo mucoperióstico, osteotomías y secciones corono radiculares también fueron asociadas al ATQ $(\mathrm{p}<0,05)$

Conclusión: factores como la edad del paciente, experiencia del cirujano, posición del molar, pericoronaritis y fusión radicular contribuyeron al ATQ.

Palabras clave: Cirugía oral, exodoncia tercer molar, tiempo quirúrgico.

\section{SUMMARY}

Introduction: Everyday, the third molar's surgery is more frequent. That fact makes necessary to know the factors that allow to anticipate the surgical time and its characteristics. The aim of this research was to know the pre-surgical and surgical factors associated with the increase of the surgical time (IST) of the mandibular third molar (3M)'s surgery.

Methodology: 145 subjects between the 15 - 40 years-old were selected from three surgical centers of Temuco (Chile). In this enclousures 158 surgeries were realized. All of the patients consulted by a 3M' s surgery, being

* Profesor Departamento de Odontología Integral. Facultad de Medicina. Universidad de La Frontera, Chile.

** Profesor Unidad de Cirugía Oral y Maxilofacial. Facultad de Odontología. Universidad Mayor Temuco,

Chile.

*** Práctica Privada. Temuco, Chile.

**** Práctica Privada. Juiz de Fora, Brasil. 
put under surgical extraction in this centers. Variables from the patients, tooth and surgery itself were studied. The information were analyzed in the Stata 9.1 statistical package, utilizing the Fisher Test and Chi-Square Test, with $\mathrm{p}<0,05$ for statistical significance.

Results: The surgical time used in the surgery was minor of 20 minutes in the $50 \%$ of the cases and minor of de 30 minutes in the $75 \%$ of the cases. The patient's age, the molar's position, the presence of pericoronaritis and the $3 \mathrm{M}^{\prime} \mathrm{s}$ root anatomy were significantly associated with a IST $(\mathrm{p}<0,05)$, of equal way the flap elevation, osteotomy and coronal or root section had statistical significance with IST $(p<0,05)$.

Conclusion: Factors like the patient's age, molar's position, surgeon's experience, pericoronaritis and the root fussion contribute significantly to and IST.

Key words: Oral surgery, third molar extraction, surgical time.

Fecha de recepción: 7 de enero de 2010.

Aceptado para publicación: 18 de febrero de 2010.

Olate S, Alister JP, Alveal R, Soto M, de Miranda Chaves Netto HD, Thomas D. Variables preoperatorias e intraoperatorias asociadas al aumento del tiempo quirúrgico en la exodoncia de terceros molares inferiores.

Av. Odontoestomatol 2012; 28 (6): 275-280.

\section{INTRODUCCIÓN}

El tercer molar (3M) se encuentra presente en el $96,6 \%$ de la población, siendo frecuente su exodoncia entre los 18 y los 25 años (1).

Las indicaciones de exodoncia del $3 \mathrm{M}$ son variadas y determinadas por diferentes factores. Gran parte de ellas responde a las necesidades del tratamiento ortodóncicos (2), donde las indicaciones específicas aún son controversiales (3). Por otra parte, la enfermedad periodontal puede ser mantenida y acentuada por la permanencia del $3 \mathrm{M}$, donde su extracción podría ayudar a la reinserción periodontal en el área distal del segundo molar (4). Dodson presentó similares conclusiones señalando además que terapias adjunta como injertos óseos en el alveolo del $3 \mathrm{M} \mathrm{o}$ regeneración ósea guiada no eran estadísticamente superiores a la exodoncia del $3 \mathrm{M}$ sin terapia adjunta (5). En pacientes sin enfermedad periodontal, las cuestiones se dirigieron al hecho de que la exodoncia podría disminuir el soporte periodontal del segundo molar; sin embargo, el estudio de revisión de Richardson y cols. (6) demostró pocos cambios en la profundidad de sondaje periodontal en el área distal del segundo molar. La habilidad, la experiencia del cirujano y la técnica quirúrgica también han sido asociadas a la reparación periodontal del segundo molar después de la exodoncia del $3 M(7)$.
La exodoncia profiláctica de $3 \mathrm{M}$ ha sido otra indicación basados en la probabilidad de generar lesiones asociadas a la permanencia del $3 \mathrm{M}$. Bohay y cols. determinaron que el $8,3 \%$ de los $3 M$ presentaba algún grado de radiolucidez radicular, lo que podría justificar su exodoncia profiláctica. Otro elemento interesante en esta línea de pensamiento esta en los cambios espaciales que desarrollaría el $3 M$ y que permitiría causar dificultades en una futura exodoncia o bien generar otro tipo de patologías (9); sin embargo, ya fue señalado también que junto con el seguimiento de pacientes con $3 \mathrm{M}$, las indicaciones de exodoncia disminuyen proporcionalmente a la complejidad de la cirugía (10).

Dentro de las características importantes a ser evaluada cuando se indica una exodoncia de $3 \mathrm{M}$ están el tiempo quirúrgico y las posibles complicaciones postoperatorias $(11,12)$. De esta forma, el tiempo quirúrgico se asocia a diferentes variables preoperatorias e intraoperatorias como la edad del paciente, grado de abertura bucal, posición del molar, tipo de inclusión, proximidad al conducto alveolar inferior, entre otros $(13,14)$. En este sentido, algunos autores han asociado el aumento del tiempo quirúrgico (ATQ) con lesiones nerviosas (14) o incluso con fracturas mandibulares; también ha sido descrito que el ATQ se asocia con extenso edema y dolor postoperatorio exacerbado $(2,11,12)$. 
El objetivo de esta investigación fue determinar las variables preoperatorias e intraoperatorias asociadas al ATQ en diferentes tipos de exodoncias de $3 M$ mandibular.

\section{MATERIAL Y MÉTODOS}

\section{Selección de pacientes}

Fue realizado un estudio descriptivo para el cual durante 1 año fueron tratados sujetos que consultaron por exodoncia de $3 \mathrm{M}$ en tres centros quirúrgicos de Temuco, Chile, correspondiendo a un centro privado (Centro Odontológico Araucanía Limitada), un centro de salud público (Consultorio Miraflores) y un centro quirúrgico universitario (Universidad de La Frontera). La muestra fue compuesta por los pacientes que cumplían con los siguientes criterios:

1. Paciente clasificado como ASAI o ASAII.

2. Ausencia de tratamiento psiquiátrico o psicológico.

3. Al menos tres meses desde el fin del periodo de lactancia.

4. Al menos tres meses de finalizado cualquier tratamiento farmacológico (ausencia de medicaciones).

5. Acceder voluntariamente al estudio junto a la firma del consentimiento informado.

Un criterio de exclusión fue la presencia de alguna patología oral compleja, por ejemplo, enfermedad periodontal agresiva, tumores maxilofaciales y trastornos temporomandibulares.

Los pacientes fueron tratados por tres especialistas en cirugía oral calibrados por un cirujano oral con 20 años de experiencia a través de 10 intervenciones quirúrgicas previas, donde los cuatro cirujanos participaron como primer cirujano, segundo cirujano u observador, permitiendo la homogeneización de los procedimientos. Fue elaborada una ficha clínica para recolectar las informaciones de las variables estudiadas, para lo cual se utilizaron las informaciones publicadas por Renton y cols. y Olmedo y cols. $(11,12)$ debido a la relación que éstas podrían presentar con el tiempo quirúrgico; de esta forma, las variables utilizadas fueron preoperatorias (edad, sexo, clasificación de Winter y morfología radicular) e intraoperatorias (colgajo, ostectomía, odontosección y com- plicaciones intraoperatorias). Las cirugías ejecutadas fueron en el $3 \mathrm{M}$ mandibular y clasificados de acuerdo a los criterios de Winter (15) mediante el análisis de radiografías panorámicas.

\section{Procedimiento quirúrgico}

El procedimiento quirúrgico fue similar al protocolo utilizado por Benediktsdóttir y cols. (16), donde se realizaron cirugías bajo anestesia local (3,6 a 5,4 ml de lidocaína al 2\% con epinefrina 1:100.000), con bloqueo al nervio alveolar inferior e infiltración de anestésico en la región vestibular del molar a extraer.

Cuando el cirujano realizo un colgajo mucoperióstico, este se extendió a través de una incisión intrasulcular vestibular en el segundo molar hasta la región sobre el tercer molar, realizando un colgajo tipo "sobre" (Figura 1). La ostectomía realizada fue con fresa de carbide cónica dentada número 702 regular (KG Sorensen ${ }^{\circledR}$ ) en baja rotación (máximo de 25.000 revoluciones por minuto) con irrigación de suero fisiológico externo. La odontosección fue realizada con fresa de carbide cónica dentada número 702 regular (KG Sorensen ${ }^{\circledR}$ ) en alta rotación (máximo de 380.000 revoluciones por minuto) e irrigación con semejantes condiciones. Las exodoncias fueron realizadas con elevadores y fórceps indicados según la situación clínica.

Tras la exodoncia, el alveolo fue inspeccionado y lavado con suero fisiológico al $0,9 \%$; la sutura fue realizada con puntos simples con hilo de seda trenzada 3-0 en aguja de $17 \mathrm{~mm}$ en 1/2 círculo. Al finalizar la cirugía, fue prescrito medicación con analgésicos, antiinflamatorios no esteroidales y antibióticos.

\section{Tiempo quirúrgico}

El tiempo quirúrgico fue medido con cronómetro iniciando la cuenta después de la fase anestésica, en el momento que fue introducido el primer instrumento quirúrgico (bisturí, elevador o fórceps) y finalizo cuando el último punto de sutura fue realizado. Las evaluaciones postquirúrgicas realizadas fueron a las $48 \mathrm{~h}$. del procedimiento y a los 7 días del mismo. 


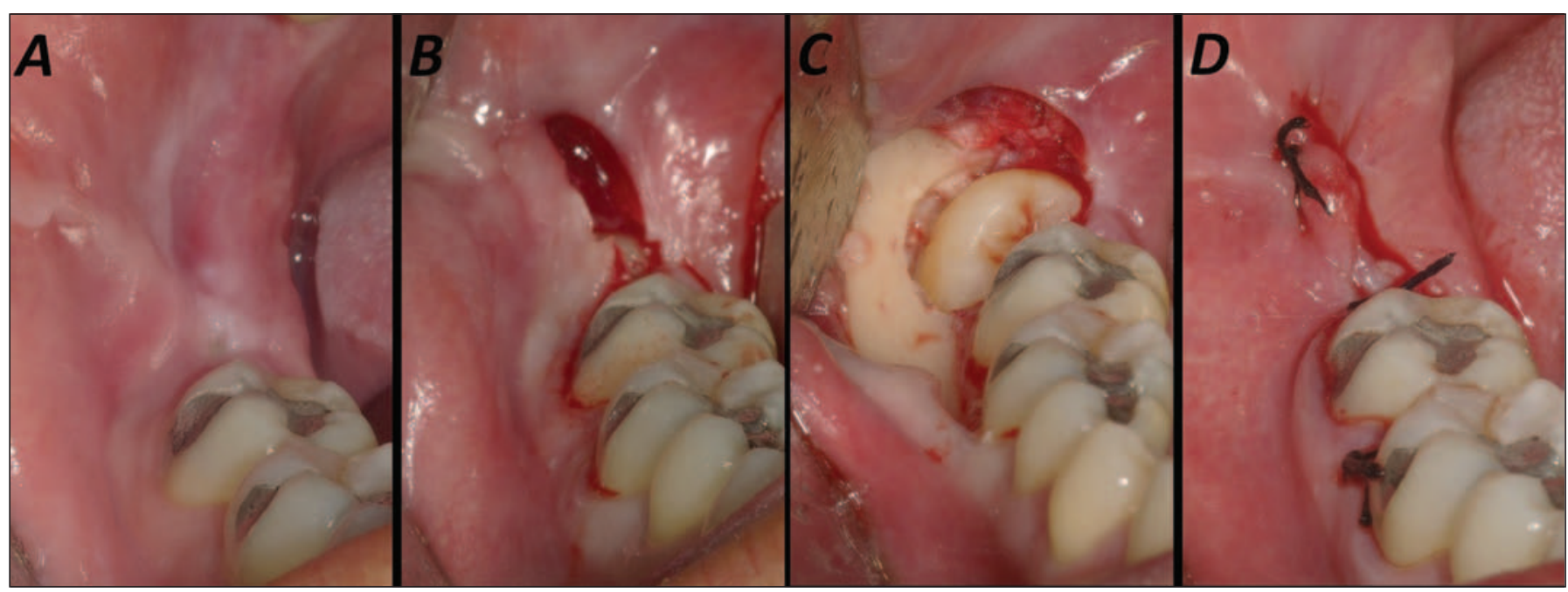

Fig. 1. Procedimiento quirúrgico para exodoncia de tercer molar inferior. A: Sitio quirúrgico con tercer molar inferior submucoso. B: Colgajo mucoperióstico de tipo "sobre" con extensión anterior hasta la papila entre primer y segundo molar. C: Ostectomía periférica realiza con fresa de baja rotación (máximo de 25.000 rpm). D: Sutura del colgajo con puntos en región posterior y en región de la papila entre primer y segundo molar.

\section{Análisis estadístico}

Los datos fueron recolectados en el software Microsoft Excel mediante tablas especialmente diseñadas; el análisis descriptivo fue realizado junto a los análisis estadísticos con las pruebas test de Fisher y chi cuadrado considerando $\mathrm{p}<0,05$ para obtener relevancia estadística.

\section{RESULTADOS}

\section{Situación sociodemográfica}

Ciento cincuenta y ocho exodoncia de $3 \mathrm{M}$ fueron realizadas en 145 sujetos en un periodo de 1 año. La edad de los pacientes fluctuó entre 15 y 45 años con una edad media de 25,11 años; la edad se asocio significativamente con el ATQ $(p=0,01)$, donde mayor edad presentaba ATQ. La relación entre hombre mujer fue de 0,56:1, presentando también una asociación estadísticamente significativa con el ATQ $(p=0,02)$.

\section{Situación preoperatoria}

El dolor preoperatorio estuvo presente en 90 de las 158 cirugías realizadas, en ausencia de una relación estadísticamente significativa entre el ATQ $(p=0,09)$; la presencia de pericoronitis previa fue observada en 46 pacientes, presentando una asociación significativa con el ATQ $(p=0,012)$. La presencia de caries en el $3 M$ no presento relación estadística con el ATQ $(p=0,024)$. El numero de raíces del $3 M$ así como la curvatura de las mismas no presento relación con el ATQ $(p=0,822)$.

\section{Situación intraoperatoria}

Como era de esperar, la ejecución de un colgajo mucoperióstico, odontosección y ostectomía fueron estadísticamente asociados con el ATQ $(p=0,000)$; las complicaciones durante la cirugía, como las fracturas dentarias no deseadas se asociaron también de forma significativa con el tiempo quirúrgico $(p=0,001)$; la ostectomía también se asocio significativamente con la presencia de complicaciones intraoperatorias $(p=0,02)$.

\section{DISCUSIÓN}

Las variables psicológicas no fueron estudiadas en nuestro trabajo; sin embargo, ya algunos autores han señalado un alto nivel de ansiedad preoperatoria generada por la exodoncia del 3M, lo cual también 
se asociaría al ATQ (17). Otro elemento no estudiado en esta investigación fue el grado de abertura bucal, que también se ha asociado al ATQ (16)

En una investigación con 496 sujetos, Slade y cols. (18) presentaron un $80 \%$ de los pacientes con una edad menor de 25 años, siendo un 59\% del sexo femenino. El $37 \%$ de la muestra presento dolor y sintomatología inflamatoria en la consulta inicial; los resultados también demostraron que individuos de mayor edad presentaban dolor asociado al $3 M$ con más frecuencia. Nuestro grupo de estudio presento una distribución semejante en términos de género y edad, existiendo cerca de un $57 \%$ con dolor preoperatorio, que fue más prevalente en sujetos mayores de 28 años, aunque sin significado estadístico.

El 49\% de las cirugías se ejecuto entre 16 y 20 minutos y cerca del $75 \%$ de las intervenciones requirió menos de 30 minutos. El estudio de Benediktsdóttir y cols. (16) demostró que el $50 \%$ de las operaciones fueron realizadas en menos que 10 minutos cuando 25 minutos fue necesario en el $90 \%$ de las cirugías. Sin embargo, en tal estudio la metodología de medición del tiempo quirúrgico no fue tan clara, sin identificar cuando iniciaba o cuando terminaba el procedimiento. Por otra parte, 14,65 minutos fue el promedio de tiempo quirúrgico utilizado por Renton y cols. (11). Susarla y cols. (19) presentaron resultados con un promedio de 6,7 minutos en un rango de tiempo de entre 0,4 y 44,3 minutos. Existe una amplia variación en los tiempos analizados sostenida principalmente por diferentes metodologías de medición; aun así, es posible identificar algunos factores que son asociadas al ATQ.

Mediante análisis univariado, Renton y cols. (11) demostraron que la edad, genero y peso del paciente, tipo de impactación del 3M, la posición horizontal del 3M, formación radicular no favorable, la proximidad al canal alveolar inferior y la experiencia del cirujano fueron relevantes en el ATQ. Benediktsdóttir y cols. (16) señalaron que la edad del paciente, la posición horizontal del 3M, la fusión radicular, la posición según la clasificación de Winter y el grado de erupción del 3M fueron relevantes en el ATQ. Nuestros resultados muestran similares resultados, destacando también que la mayor edad del paciente y el sexo masculino fueron asociados con el ATQ; la presencia de dolor preoperatorio y la posición poco favorable se presentaron cercanas a obtener alguna relación estadística con el ATQ.

Evidentemente, cualquier procedimiento realizado durante la cirugía como la presencia de un colgajo mucoperióstico, ostectomía y odontosección conlleva un ATQ; sin embargo, es interesante el hecho de que la necesidad de ostectomías presento una relación significativa con la presencia de complicaciones durante la cirugía como fracturas dentarias no deseadas o dolor intraoperatorio. Podemos especular que exodoncias que necesitan de ostectomías son procedimientos más complejos, haciendo posible mayor ansiedad en el paciente y un difícil manejo intraoperatorio.

\section{CONCLUSIÓN}

Finalmente, podemos concluir que factores como la edad y el género del paciente junto a una posición del $3 \mathrm{M}$ poco favorable y raíces fusionadas se relacionan con el ATQ en exodoncias de $3 \mathrm{M}$ mandibular.

\section{BIBLIOGRAFÍA}

1. Kruger E, Thomson WM, Konthasinghe P. Third molar outcomes from age 18 to 26: findings from a population - based New Zealand longitudinal study. Oral Surg Oral Med Oral Pathol Radiol Endod 2001;92:150-5.

2. Chaparro-Avendaño A, Pérez-García S, Valmaseda-Castellón E, Berini-Aytés L, Gay-Escoda C. Morbidity of third molar extraction in patients between 12 and 18 years of age. Med Oral Patol Oral Cir Bucal 2005;10:422-31.

3. Harradine N, Pearson M, Toth B. The effect of extraction of third molars on late lower incisor crowding: a randomized controlled trial. $\mathrm{Br} \mathrm{J}$ Orthodontics 1998;25:117-22.

4. Krausz AA, Machtei EE, Peled M. Effects of lower third molar extraction on attachment level and alveolar bone height of the adjacent second 
molar. Int J Oral Maxillofac Surg 2005;34:75660.

5. Dodson T. Management of mandibular third molar extraction sites to prevent periodontal defects. J Oral Maxillofac Surg 2004;62:121324.

6. Richardson D, Dodson T. Risk of periodontal defects alter third molar surgery: an exercise in evidence - based clinical decisión - making. Oral Surg Oral Med Oral Pathol Radiol Endod 2005;100:133-7.

7. Chang H-H, Lee J-J, Kok S-H, Yang P-J. Periodontal healing after mandibular third molar surgery - a comparision of distolingual alveolectomy and tooth division techniques. Int $\mathrm{J}$ Oral Maxillofac Surg. 2004;33:32-7.

8. Bohay R, Mara T, Sawula K, Lapointe H. A preliminary radiographic study of mandibular para-radiular third molar radiolucencies. Oral Surg Oral Med Oral Pathol Oral Radiol Endod 2004;98:97-101.

9. Ventä I, Turtola L, Ylipaavalniemi P. Radiographic follow-up of impacted third molar from age 20 to 32 years. Int J Oral Maxillofac Surg 2001;30: 54-7.

10. Vënta I, Ylipaavalniemi P, Tórtola L. Long - term evaluation of estimates of need for third molar removal. J Oral Maxillofac Surg 2000;58:288-91.

11. Renton T, Smeeton N, McGurk M. Factors predictive of difficulty of mandibular third molar surgery. Br Dental J 2001;190:607-10

12. Olmedo MV, Vallecillo M, Gálvez. Relation of patient and surgical variables to postoperative pain and inflammation in the extraction $\mathrm{f}$ third molars. Med Oral Patol Oral Cir Bucal 2002; 7: 360-9.
13. Susarla S, Blaeser B, Magalnick D. Third molar surgery and associated complications. Oral Maxillofac Clin N Am 2003;15:177-86.

14. Hull D, Shugars D, White R, Phillips C. Proximity of a low third molar to the inferior alveolar canal as a predictor of delayed recovery. J Oral Maxillofac Surg 2006;64:371-6.

15. Winter GB. Principles of exodontia as applied to the impacted third molar. St. Louis, American Medical Books, 1926.

16. Benediktsdóttir I, Wenzel A, Petersen J, Hintze H. Mandibular third molar removal: risk indicators for extended operation time, postoperative pain, and complications. Oral Surg Oral Med Oral Pathol Oral Radiol Endod. 2004;97:438-46.

17. Lago-Méndez L, Diniz-Freitas M, Senra-Rivera C, Seoane Pesqueira G, Gándara-Rey JM, GarciaGarcia A. Dental anxiety befote removal of a third molar and association with general trait anxiety. J Oral Maxillofac Surg 2006;64:1404-8.

18. Slade G, Foy S, Shugars D, Phillips C, White R. The impact of third molar symptoms, pain, and swelling on oral health - relates quality of life. J Oral Maxillofac Surg 2004; 62:1118-24.

19. Susarla S, Dodson T. Risk factors for third molar extraction difficulty. J Oral Maxillofac Surg. 2004; 62:1363-71.

\section{CORRESPONDENCIA}

Dr. Sergio Olate

Departamento de Odontología Integral

Facultad de Medicina

Universidad de La Frontera

Manuel Montt, 112, Piso $4^{\circ}$.

Temuco, Chile

E-mail: solate@ufro.cl 